\title{
Complexity of limit-cycle problems in Boolean networks
}

\author{
Florian Bridoux ${ }^{1}$, Caroline Gaze-Maillot ${ }^{1}$, Kévin Perrot ${ }^{1,2}$, and Sylvain Sené ${ }^{1}$ \\ ${ }^{1}$ Aix Marseille Univ., Univ. Toulon, CNRS, LIS, UMR 7020, Marseille, France. \\ ${ }^{2}$ Univ. Côte d'Azur, CNRS, I3S, UMR 7271, Sophia Antipolis, France.
}

\begin{abstract}
Boolean networks are a general model of interacting entities, with applications to biological phenomena such as gene regulation. Attractors play a central role, and the schedule of entities update is a priori unknown. This article presents results on the computational complexity of problems related to the existence of update schedules such that some limit-cycle lengths are possible or not. We first prove that given a Boolean network updated in parallel, knowing whether it has at least one limit-cycle of length $k$ is NP-complete. Adding an existential quantification on the block-sequential update schedule does not change the complexity class of the problem, but the following alternation brings us one level above in the polynomial hierarchy: given a Boolean network, knowing whether there exists a block-sequential update schedule such that it has no limit-cycle of length $k$ is NP ${ }^{N P}$-complete.
\end{abstract}

\section{Introduction}

Boolean networks (BNs) were introduced by McCulloch and Pitts in the 1940s through the well known formal neural networks [17] that are specific BNs governed by a multidimensional threshold function. Informally, BNs are finite dynamical systems in which entities having Boolean states may interact with each other over discrete time. After their introduction, neural networks were studied in depth from the mathematical standpoint. Among the main works on them are the introduction by Kleene of finite automata and regular expression [16], first results on the dynamical behaviors of linear feedback shift register [13] and linear networks [8]. These researches led Kauffman and Thomas (independently) from the end of the 1960s to develop the use of BNs in the context of biological networks modeling [15, 27], which has paved the way to numerous applied works at the interface between molecular biology, computer science and discrete mathematics. In parallel, theoretical developments were done in the framework of linear algebra and numerical analysis by Robert [24, and in that of dynamical system theory and computational models, which constitutes the lens through which we look at BNs in this paper.

In this context, numerous studies have already been led and have brought very important results. Considering that a BN can be defined as a collection of local Boolean 
functions (each of these defining the discrete evolution of one entity over time given the states of the entities that influence it), it can be represented by a directed graph at the static level, classically called the interaction digraph. Moreover, as a BN is by definition of finite size here, it is trivial to see that the trajectory of any of its configurations (or global state) ends into a cycle that can be a fixed point or a limit-cycle. The main theoretical objective in the domain is twofold: obtaining (combinatorial or algebraic) characterizations of the dynamics of such objects, through either their definition as collections of Boolean functions or their interaction graphs, and understanding the complexity of finding such characterizations.

In these lines, Robert showed that retroaction cycles between entities in the interaction graph are necessary for a BN to have a non-trivial dynamical behavior [25] and Thomas conjectured strong relations between these retroaction cycles (well known as positive and negative cycles) and the existence of multi-stationarity (several fixed points) or limit-cycles [28] which were proven later [21, 23, 22]. A notable fact about these seminal works is that they underline clearly that retroaction cycles are the engines of behavioral complexity (or dynamical richness). More recently, a real effort has been impulsed on the understanding of retroaction cycles. In particular, Demongeot et al. characterized exhaustively the behaviors of retroaction cycles and some of their intersections [7]. Furthermore, the problem of counting the number of fixed points and limit-cycles has mushroomed. Advances have been done concerning fixed points [3, 5]. Nevertheless, due to the high dependence of limit-cycle appearance according to the update schedule (i.e. the way / order under which entities are updated over time), no general combinatorial results have been obtained, except for retroaction cycles [7]. In relation to complexity theory, the main known results based on $\mathrm{BNs}$ are: determining if a BN admits fixed points is NP-complete, counting fixed points is \#P-complete [9, 19, determining if a fixed point has a non-trivial attraction basin is NP-complete, determining if there exists another update schedule that conserves the limit-cycles of a given BN evolving in parallel is NP-hard [4]. Moreover, a recent work [6] focused on related questions on fixed point complexity by focusing on interaction digraphs and not on BNs anymore (notice that several BNs admit the same interaction digraph).

In this paper, we impregnate from these last results and transfer the problematics to limit-cycles, which constitutes to our knowledge one of the first attempts to understanding limit-cycles from the complexity theory point of view with [4, 12. More precisely, considering that the input is a $\mathrm{BN}$, we prove that determining if a $\mathrm{BN}$ evolving admits a limit-cycle of length $k$ is NP-complete whatever the update schedule (in the class of block-sequential updating modes, that is updating modes defined as ordered partitions of the set of entities). Furthermore, we show that determining if there exists a blocksequential update schedule such that a given BN admits no limit-cycles of length $k$ is $\mathrm{NP}^{\mathrm{NP}}$-complete.

In what follows, Section 2 presents the main definitions that are used in the paper. Section 3 gives a brief state of the art of the problematic addressed. The main results of the paper are given in Section 4 and are followed by a conclusion developing some perspectives of this work. 


\section{Definitions}

We denote $\mathbb{N}_{+}$the set of strictly positive integers, and $[n]=\{1, \ldots, n\}$ for some $n \in \mathbb{N}_{+}$. For $x \in\{0,1\}^{n}$ and $i \in[n]$, we denote $x_{i}$ the component $i$ of $x$, and $x+e_{i}$ the vector of $\{0,1\}^{n}$ obtained by flipping component $i$ of $x$ (addition is performed modulo 2). The symbol $\oplus$ is used for the binary operator exclusive or (xor).

\subsection{Boolean networks}

A Boolean network (BN) is a function $f:\{0,1\}^{n} \rightarrow\{0,1\}^{n}$, that we see as $n$ local functions $f_{1}, \ldots, f_{n}$ with $f_{i}:\{0,1\}^{n} \rightarrow\{0,1\}$ for each $i \in[n]$. The interaction digraph of a BN $f$ captures the actual dependencies among its components, and is defined as $G_{f}=(V, A)$, with $V=[n]$ and

$$
(i, j) \in A \quad \Longleftrightarrow \quad \exists x \in\{0,1\}^{n}: f_{j}(x) \neq f_{j}\left(x+e_{i}\right) .
$$

The arcs of the interaction digraph may be assigned signs $\sigma: A \rightarrow\{+,-, \pm\}$ as follows:

- $\sigma(i, j)=+$ when $\exists x \in\{0,1\}^{n}: x_{i}=0 \wedge f_{j}(x)>f_{j}\left(x+e_{i}\right)$,

- $\sigma(i, j)=-$ when $\exists x \in\{0,1\}^{n}: x_{i}=0 \wedge f_{j}(x)<f_{j}\left(x+e_{i}\right)$,

- $\sigma(i, j)= \pm$ when both conditions above hold.

For convenience, we may use various symbols to denote the components of the network, but as it will always be a finite set a bijection with $[n]$ is straightforward. The size of a $\mathrm{BN}$ is its number of components.

\section{$2.2 \quad$ Update schedules}

The configuration space is $\{0,1\}^{n}$, and it remains to explain how components are updated. Given a BN $f$, a configuration $x$ and a subset $I \subseteq[n]$, we $\operatorname{denot} e^{1} f^{(I)}(x)$ the configuration obtained by updating components of $I$ only, i.e.

$$
\text { for any } i \in[n], \quad f^{(I)}(x)_{i}= \begin{cases}f_{i}(x) & \text { if } i \in I \\ x_{i} & \text { otherwise. }\end{cases}
$$

Remark that $f^{([n])}=f$. A block-sequential update schedule is an ordered partition of $[n]$, denoted $W=\left(W_{1}, \ldots, W_{t}\right)$, and a BN $f$ updated according to $W$ gives the deterministic discrete dynamical system on $\{0,1\}^{n}$ defined as

$$
f^{(W)}=f^{\left(W_{t}\right)} \circ \cdots \circ f^{\left(W_{2}\right)} \circ f^{\left(W_{1}\right)} .
$$

The update schedule $([n])$ is called parallel (or synchronous).

\footnotetext{
${ }^{1}$ Parenthesis are used to differentiate update schedules from iterations of a function.
} 


$$
\text { (1) (2) } \begin{array}{ll}
f_{1}(x)=x_{2} \\
f_{2}(x)=x_{1}
\end{array}
$$

Figure 1: Two BNs and their respective interaction digraphs (all arcs are positive). Left: for $W=(\{1\},\{2\})$ we have $\phi_{2}\left(f^{(W)}\right)=0$, whereas for the parallel mode we have $\phi_{2}(f)=1$. Right: for $W^{\prime}=(\{1\},\{2,3\})$ we have $\phi_{2}\left(f^{\prime\left(W^{\prime}\right)}\right)=1$ with $001 \leftrightarrow 110$, whereas for the parallel mode we have $\phi_{2}\left(f^{\prime}\right)=0$.

\subsection{Attractors}

Given that the configuration space is finite and the dynamics is deterministic, the orbit of any configuration convergences to a fixed point (a configuration $x$ such that $f^{(W)}(x)=x$ ) or to a limit-cycle (a configuration $x$ such that $\left(f^{(W)}\right)^{k}(x)=x$ for some length $k \in \mathbb{N}_{+}$, and such that $\left(f^{(W)}\right)^{\ell}(x) \neq x$ for any $\left.\ell \in[k-1]\right)$. A fixed point is a limit-cycle of length one, a limit-cycle is assimilated to any of its configurations, and has a unique length.

Given a BN $f$, an update schedule $W$, and $k \in \mathbb{N}_{+}$, we denote $\Phi_{k}\left(f^{(W)}\right)$ the set of configurations in limit-cycles of length $k$, i.e.

$$
\Phi_{k}\left(f^{(W)}\right)=\left\{x \in\{0,1\}^{n} \mid\left(f^{(W)}\right)^{k}(x)=x \text { and } \forall 1 \leq \ell<k,\left(f^{(W)}\right)^{\ell}(x) \neq x\right\}
$$

and $\phi_{k}\left(f^{(W)}\right)=\frac{\left|\Phi_{k}\left(f^{(W)}\right)\right|}{k}$ the number of limit-cycles of length $k$. Remark that for a fixed $k$, the quantity $\phi_{k}\left(f^{(W)}\right)$ may vary depending on $W$ (see Figure 1 ).

For retroaction cycles (such as those of Figure 1), the dynamical behavior in terms of number of limit-cycles of size $k$, whatever the update schedule, is entirely characterized in [7] on the basis of [1].

\subsection{Problems}

Remark 1. Note that an input BN $f$ is encoded with its local functions as propositional formulas (see also Remark 3 at the end).

We are interested in the following decision problems related to attractors in the dynamics of BNs, and especially limit-cycles.

\section{$k$-limit-cycle problem $(k$-LC)}

Input: a BN $f$ updated in parallel.

Question: does $\phi_{k}(f) \geq 1$ ?

Remark 2. $f$ updated in parallel is not a limitation here, since one can transform in polynomial time a $B N f$ and an updated schedule $W$ into a $B N f^{\prime}$ updated in parallel such that $f^{\prime}=f^{(W)}$ (simply construct local functions of $f^{\prime}$ from those of $f$ and $W$ ), as presented in [26]. 


\section{Block-sequential $k$-limit-cycle problem (BS $k$-LC)}

Input: a $\mathrm{BN} f$.

Question: does there exist $W$ block-sequential such that $\phi_{k}\left(f^{(W)}\right) \geq 1$ ?

\section{Block-sequential no $k$-limit-cycle problem (BS no $k$-LC)}

Input: a $\mathrm{BN} f$.

Question: does there exist $W$ block-sequential such that $\phi_{k}\left(f^{(W)}\right)=0$ ?

Fixed points are invariant over block-sequential update schedules [10], consequently 1LC and BS 1-LC are identical. However, the last two problems are not complement of each other, because there exist some instance positive in both (see Figure 1 for an example).

For the reductions giving complexity lower bounds, we need the following classical problems. For a formula $\psi$ on $\left\{\lambda_{1}, \ldots, \lambda_{n}\right\}$ and a partial assignment $v:\left\{\lambda_{1}, \ldots, \lambda_{s}\right\} \rightarrow$ $\{0,1\}$ for some $s \in[n]$, we denote $\psi[v]$ the substitution $\psi\left[\lambda_{1} \leftarrow v\left(\lambda_{1}\right), \ldots, \lambda_{s} \leftarrow v\left(\lambda_{s}\right)\right]$.

\section{3-SAT}

Input: a 3 -CNF formula $\psi$ on $\left\{\lambda_{1}, \ldots, \lambda_{n}\right\}$.

Question: is $\psi$ satisfiable?

\section{$\exists \forall$-3-SAT}

Input: a 3 -CNF formula $\psi$ on $\left\{\lambda_{1}, \ldots, \lambda_{n}\right\}$ and $s \in[n]$.

Question: is there an assignment $v$ of $\lambda_{1}, \ldots, \lambda_{s}$ such that

all assignments of $\lambda_{s+1}, \ldots, \lambda_{n}$ satisfy $\psi[v]$ ?

3-SAT is a well known NP-complete problem [14], and $\exists \forall$-3-SAT is NPNP -complete [20] (one level above in the polynomial hierarchy). Also, note that NPNP $=$ NPco-NP since an oracle language or its complement are equally useful.

\section{State of the art}

The $k$-limit-cycle problem is known to be NP-complete for $k=1$ [9], and the fixed points of a $\mathrm{BN}$ are invariant for any block-sequential update schedule [10. It has been proven in [2] that given a $\mathrm{BN} f$, it is NP-complete to know whether there exist two blocksequential update schedules $W, W^{\prime}$ such that $f^{(W)} \neq f^{\left(W^{\prime}\right)}$ (that is, they differ on at least one configuration). This problem is indeed surprisingly difficult, but the proof relies on a basic construction similar to Theorem 1 for $k=1$. More over, in [4, the authors study the computational complexity of limit cycle problems. Given a $\mathrm{BN} f$, an update schedule $W$ and a limit-cycle $C$ of $f^{(W)}$, it is NP-complete to know whether there exists another update schedule $W^{\prime}$ (not equivalent to $W$ ) such that $f^{(W)}$ also has the limit-cycle $C$. Some variants of this problem are deduced to be NP-complete: knowing whether the sets of limit cycles are equal, and whether the sets of limit-cycles share at least one element. This work focuses on finding block-sequential update schedules sharing limit cycles. After writing this article, we learned that the PhD thesis of Gómez [12] contains results of a very close flavor: given a $\mathrm{BN} f$, determining whether it is possible to find a 
block-sequential update schedule $W$ such that $f^{(W)}$ has at least one limit cycle (of any length greater than two) is NP-complete, even when restricted to AND-OR networks. Moreover, the problem of finding a block-sequential $W$ such that $f^{(W)}$ has only fixed points is NP-hard. In the sequel, we prove an analogous bound for the existence problem (Corollary 1. Our construction also has only AND-OR local functions), and a stronger tight bound for the non-existence problem (Theorems 3, 4 and Corollary 22). As a difference, in our setting the length of the limit-cycle is fixed in the problem definition. It is also proven in [12] that given a $\mathrm{BN} f$ and two configurations $x, y$, is there a $W$ such that $f^{(W)}(x)=y$ ? is an NP-complete problem.

Eventually, questions on the maximum number of fixed points possible when only the interaction digraph of a $\mathrm{BN}$ is provided, have already let some complexity classes higher than NP appear in problems related to the attractors of BNs [6].

\section{Complexity of limit-cycle problems}

The constructions presented in this section are gradually extended with more involved arrangements of components, to prove complexity lower bounds from formula satisfaction problems. The first result adapts a folklore proof for fixed points (case $k=1$ ).

Theorem 1. $k$-LC is NP-complete for any $k \in \mathbb{N}_{+}$.

Proof. The problem belongs to NP because one can check in polynomial time a certificate consisting of one configuration $x \in\{0,1\}^{n}$ of the limit-cycle of length $k$. Indeed, to check that $x \in\{0,1\}^{n}$ is in a limit-cycle of size $k$, it is sufficient to check that $f(x), \ldots, f^{k-1}(x)$ are different from $x$ and that $f^{k}(x)$ equals $x$.

To show that it is NP-hard, we present a reduction from 3-SAT. Given a 3-CNF formula $\psi$ on $\left\{\lambda_{1}, \ldots, \lambda_{n}\right\}$ with $m$ clauses $C_{1}, \ldots, C_{m} \in\left(\left\{\lambda_{1}, \ldots, \lambda_{n}\right\} \cup\left\{\neg \lambda_{1}, \ldots, \neg \lambda_{n}\right\}\right)^{3}$, we construct the following $\mathrm{BN}$ of size $n+m+k$. The components are

$$
\left\{\lambda_{1}, \ldots, \lambda_{n}\right\} \cup\left\{C_{1}, \ldots, C_{m}\right\} \cup\left\{\psi_{1}, \ldots, \psi_{k}\right\}
$$

and the local functions are

- $f_{\lambda_{i}}(x)=x_{\lambda_{i}}$ for $i \in[n]$,

- $f_{C_{j}}(x)=\bigvee_{\lambda_{i} \in C_{j}} x_{\lambda_{i}} \vee \bigvee \neg \lambda_{i} \in C_{j} \neg x_{\lambda_{i}}$ for $j \in[m]$,

- $f_{\psi_{1}}(x)=\neg x_{\psi_{1}} \wedge x_{\psi_{k}} \wedge\left(x_{C_{1}} \wedge \cdots \wedge x_{C_{m}}\right)$,

- $f_{\psi_{i}}(x)=\neg x_{\psi_{i}} \wedge x_{\psi_{i-1}}$ for $i \in\{2, \ldots, k\}$.

If $k=1$, then we set $f_{\psi_{1}}(x)=\neg x_{\psi_{1}} \vee\left(x_{C_{1}} \wedge \cdots \wedge x_{C_{m}}\right)$. An example signed interaction digraph of this $\mathrm{BN}$ is presented on Figure 2 .

The idea is that to get a limit-cycle of length $k$, one is forced to find in $x_{\lambda_{1}}, \ldots, x_{\lambda_{n}}$ an assignment satisfying $\psi$, in order to have $x_{C_{j}}=1$ for all $j \in[\mathrm{m}]$ and a configuration cycling through $x_{\phi_{1}}, \ldots, x_{\phi_{k}}$. Otherwise if $x_{\lambda_{1}}, \ldots, x_{\lambda_{n}}$ does not satisfy $\psi$, then the attractor is a fixed point (except for the case $k=1$ ). The articulation between the formula assignment and the limit-cycle of length $k$ hinges upon $f_{\psi_{1}}$. 


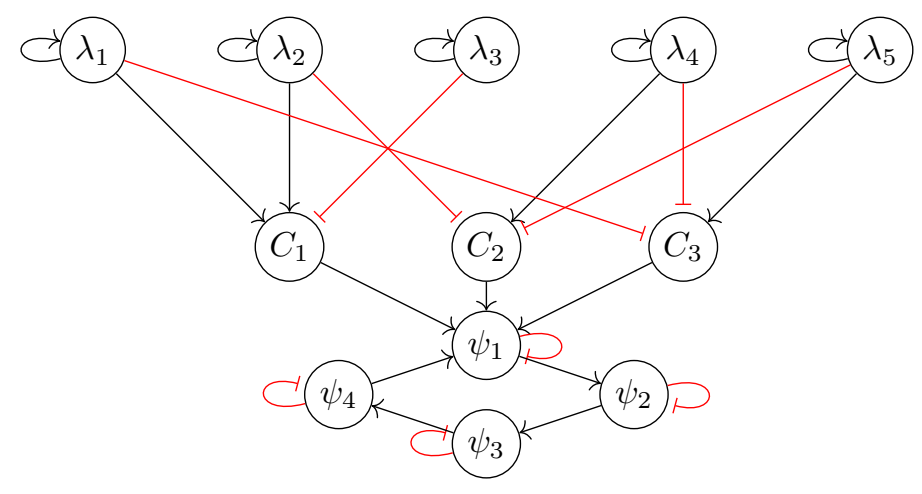

Figure 2: Signed interaction digraph of the BN obtained for $k=4$ and the 3-CNF formula $\psi=\left(\lambda_{1} \vee \lambda_{2} \vee \neg \lambda_{3}\right) \wedge\left(\neg \lambda_{2} \vee \lambda_{4} \vee \neg \lambda_{5}\right) \wedge\left(\neg \lambda_{1} \vee \neg \lambda_{4} \vee \lambda_{5}\right)$. Negative arcs (-) are red with a flat head, positive arcs $(+)$ are black (there are no \pm arcs).

Let us now prove that $\psi$ is satisfiable if and only if the $\mathrm{BN}$ has a limit-cycle of length $k$. Suppose $\psi$ is satisfied for $v:\left\{\lambda_{1}, \ldots, \lambda_{n}\right\} \rightarrow\{0,1\}$, then the following configuration $x \in\{0,1\}^{n+m+k}$ is part of a limit-cycle of length $k$ :

- $x_{\lambda_{i}}=v\left(\lambda_{i}\right)$ for all $i \in[n]$,

- $x_{C_{j}}=1$ for all $j \in[\mathrm{m}]$,

- $x_{\psi_{1}}=1$ and $x_{\psi_{2}}=\cdots=x_{\psi_{k}}=0$.

Indeed, the state of components $\left\{\lambda_{1}, \ldots, \lambda_{n}\right\} \cup\left\{C_{1}, \ldots, C_{m}\right\}$ do not change, and the unique state 1 in the cycl ${ }^{2}$ of components $\left\{\psi_{1}, \ldots, \psi_{k}\right\}$ moves one component forward at each step (all other components being in state 0 ), and comes back to the initial configuration $x$ in $k$ steps, i.e. $f^{k}(x)=x$.

For the reverse direction, suppose there is a limit-cycle of length $k$, and let $x$ be one of its configurations. Remark that in any attractor, the states of components $\left\{\lambda_{1}, \ldots, \lambda_{n}\right\}$ are fixed, and so are the states of components $\left\{C_{1}, \ldots, C_{m}\right\}$. As a consequence, in the local function $f_{\psi_{1}}$, the evaluation of the part $\left(x_{C_{1}} \wedge \cdots \wedge x_{C_{m}}\right)$ is fixed. For the sake of contradiction suppose that it is evaluated to 0 , then so is $x_{\psi_{1}}$, then so is $x_{\psi_{2}}$, etc, and $x$ is a fixed point (in the case $k=1$ we have $f_{\psi_{1}}(x) \neq x_{\psi_{1}}$ ). Therefore, components $\left\{C_{1}, \ldots, C_{m}\right\}$ are all in state 1 , which, according to their local functions, is possible if and only if each of them has at least one of its predecessors in state 1 if it appears positively in the corresponding clause, or in state 0 if it appears negatively. As a conclusion the states of components $\left\{\lambda_{1}, \ldots, \lambda_{n}\right\}$ in $x$ correspond to a valuation satisfying $\psi$.

The second result initiates the consideration of update schedules in complexity studies of the dynamics of BNs. However, with an additional existential quantification on the update schedule the problem remains NP-complete (there was already an existential quantification on configurations for the existence of a limit-cycle), and it turns out that the same construction proves it.

${ }^{2}$ The cycle in the interaction digraph. 
Corollary 1. BS $k$-LC is NP-complete for any $k \in \mathbb{N}_{+}$.

Proof. This problem still belongs to the class NP, as one can check in polynomial time a certificate consisting of a block-sequential $W$ on $[n]$ and one configuration $x \in\{0,1\}^{n}$ of the limit-cycle of length $k$. Indeed, it is sufficient to check that $f^{(W)}(x), \ldots,\left(f^{(W)}\right)^{k-1}(x)$ are different from $x$ and that $\left(f^{(W)}\right)^{k}(x)$ equals $x$.

For the NP-hardness we use the same construction as in the proof of Theorem 1. Indeed, remark that the existential quantification on a block-sequential update schedule fits the reasoning. For the left to right direction of the if and only if we use the same $x$ with $W=[n]$. And for the reverse direction, if $\psi$ is not satisfiable then for any blocksequential update schedule any configuration converges to a fixed point (the upper part is always fixed, and $x_{\psi_{1}}=0$ fixes the cycle).

We have seen in Theorem 1 and Corollary 1 that with two consecutive existential quantifications (one for a block-sequential update schedule and one for a configuration of a limit-cycle) the problem remains in NP. However, BS no $k$-LC corresponds to an existential quantification (for a block-sequential update schedule) followed by a universal quantification (for the absence of a limit-cycle). The next results therefore jump one level above in the polynomial hierarchy.

Theorem 2. BS no $k$-LC is in NPN for any $k \in \mathbb{N}_{+}$.

Proof. The problem belongs to the class NPco-NP $=$ NPNP, as one can guess nondeterministically a block-sequential update schedule $W$ and then check in polynomial time (in NP), using an oracle in co-NP, whether $\phi_{k}\left(f^{(W)}\right)=0$. Once $W$ is fixed this last question is indeed in co-NP, as it is the complement of $\mathbf{k - L C}$, see Remark 2 and Theorem 1 .

The hardness proof is splitted into three results, developing some incremental mechanisms and constructions.

Theorem 3. BS no $k$-LC is NPNP-hard for all $k$ even and strictly greater than 2.

Proof. We present a reduction from $\exists \forall$-3-SAT. Given a 3 -CNF formula $\psi$ on $\left\{\lambda_{1}, \ldots, \lambda_{n}\right\}$ with $m$ clauses denoted as usual $C_{1}, \ldots, C_{m}$, and an integer $s \in[n]$, we construct the following $\mathrm{BN}$ of size $2 s+n+m+k+2$. The components are

$$
\{\Omega, \psi\} \cup\left\{\lambda_{1}, \ldots, \lambda_{n}\right\} \cup\left\{\lambda_{1}^{\prime}, \ldots, \lambda_{s}^{\prime}\right\} \cup\left\{\lambda_{1}^{\prime \prime}, \ldots, \lambda_{s}^{\prime \prime}\right\} \cup\left\{C_{1}, \ldots, C_{m}\right\} \cup\left\{\psi_{0}, \ldots, \psi_{k-1}\right\}
$$

and the local functions are

- $f_{\Omega}(x)=\neg x_{\Omega}$,

- $f_{\lambda_{i}^{\prime}}(x)=f_{\lambda_{i}^{\prime \prime}}(x)=x_{\Omega}$ for $i \in[s]$,

- $f_{\lambda_{i}}(x)=x_{\lambda_{i}^{\prime}} \oplus x_{\lambda_{i}^{\prime \prime}}$ for $i \in[s]$, and $f_{\lambda_{i}}(x)=x_{\lambda_{i}}$ for $i \in[n] \backslash[s]$,

- $f_{C_{j}}(x)=\bigvee_{\lambda_{i} \in C_{j}} x_{\lambda_{i}} \vee \bigvee \neg \lambda_{i} \in C_{j} \neg x_{\lambda_{i}}$ for $j \in[m]$,

- $f_{\psi}(x)=x_{C_{1}} \wedge \cdots \wedge x_{C_{m}}$,

- if $i \in\{0, \ldots, k-1\}$ is even then $f_{\psi_{i}}(x)=\left\{\begin{array}{ll}x_{\psi_{i}} & \text { if } x_{\psi}=1 \vee x_{\Omega}=0 \\ x_{\psi_{i-1} \bmod k} & \text { otherwise }\end{array}\right.$, 


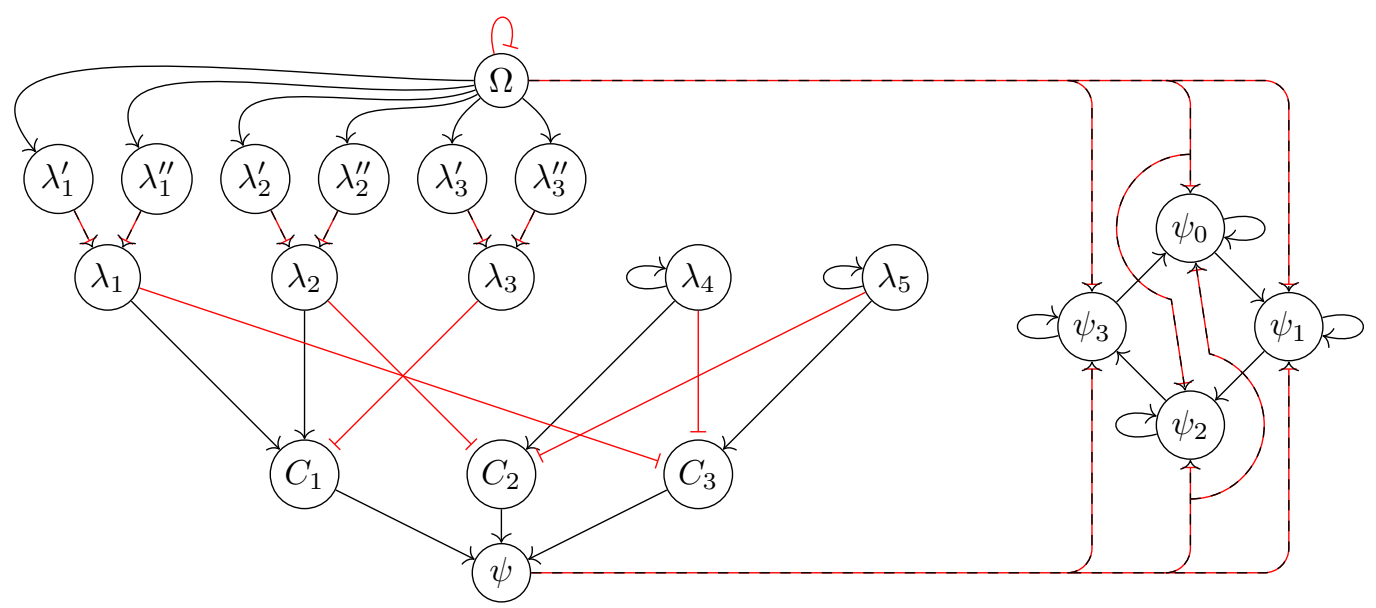

Figure 3: Signed interaction digraph of the BN obtained for $k=4$, the 3-CNF formula $\psi=\left(\lambda_{1} \vee \lambda_{2} \vee \neg \lambda_{3}\right) \wedge\left(\neg \lambda_{2} \vee \lambda_{4} \vee \neg \lambda_{5}\right) \wedge\left(\neg \lambda_{1} \vee \neg \lambda_{4} \vee \lambda_{5}\right)$, and $s=3$. Negative arcs $(-)$ are red with a flat head, positive arcs $(+)$ are black, positive-negative arcs $( \pm)$ are dashed with both colors and heads. Components $\Omega$ and $\psi$ are both connected to components $\psi_{0}, \psi_{1}, \psi_{2}$ and $\psi_{3}$ with arcs of sign \pm .

- if $i \in\{0, \ldots, k-1\}$ is odd then $f_{\psi_{i}}(x)=\left\{\begin{array}{ll}x_{\psi_{i}} & \text { if } x_{\psi}=1 \vee x_{\Omega}=1 \\ x_{\psi_{i-1}} & \text { otherwise }\end{array}\right.$.

An example signed interaction digraph of this BN is presented on Figure 3 .

The idea is that to prevent a possible limit-cycle of length $k$ to take place on components $\left\{\psi_{0}, \ldots, \psi_{k-1}\right\}$, one is forced to solve the $\forall \exists$-3-SAT instance and let $x_{\psi}=1$ in any configuration $x$ that is part of an attractor. The existential variables are assigned in the block-sequential update schedule (on the updates of $\lambda_{i}, \lambda_{i}^{\prime}$ and $\lambda_{i}^{\prime \prime}$ relative to the update of $\Omega$, for $i \in[s]$ ), and the universal variables all appear in both states in attractors (thanks to the positive loops on components $\left\{\lambda_{s+1}, \ldots, \lambda_{n}\right\}$ ).

Let us now prove that there exists an assignment $v:\left\{\lambda_{1}, \ldots, \lambda_{s}\right\} \rightarrow\{0,1\}$ such that all assignments $v^{\prime}:\left\{\lambda_{s+1}, \ldots, \lambda_{n}\right\} \rightarrow\{0,1\}$ verify $\psi[v]\left[v^{\prime}\right] \equiv 1$, if and only if there exists a block-sequential update schedule $W$ such that $f^{(W)}$ has a no limit-cycle of length $k$. Suppose there exists such an assignment $v$, then we define

$$
W=\left(T^{\prime},\{\Omega\}, F^{\prime} \cup\left\{\lambda_{1}^{\prime \prime}, \ldots, \lambda_{s}^{\prime \prime}\right\},\left\{\lambda_{1}, \ldots, \lambda_{n}\right\} \cup\left\{C_{1}, \ldots, C_{m}\right\} \cup\{\psi\} \cup\left\{\psi_{0}, \ldots, \psi_{k-1}\right\}\right)
$$

with $T^{\prime}=\left\{\lambda_{i}^{\prime} \mid v\left(\lambda_{i}\right)=1\right\}$ and $F^{\prime}=\left\{\lambda_{i}^{\prime} \mid v\left(\lambda_{i}\right)=0\right\}$ (for $i \in[s]$ ). We claim that $f^{(W)}$ has no limit-cycle of length $k$. Indeed, the state of components $\left\{\lambda_{1}, \ldots, \lambda_{s}\right\}$ correspond to the valuation $v$, because $\lambda_{i}^{\prime}$ and $\lambda_{i}^{\prime \prime}$ for positive (resp. negative) variables are updated before and strictly after (resp. both strictly after) component $\Omega$ flips his state when it is updated, therefore are equal (resp. not equal) when local functions $f_{\lambda_{i}}$ compute their xor. The states of components $\{\Omega\} \cup\left\{\lambda_{1}^{\prime}, \ldots, \lambda_{s}^{\prime}\right\} \cup\left\{\lambda_{1}^{\prime \prime}, \ldots, \lambda_{s}^{\prime \prime}\right\}$ all flip at each step (hence the required conditions on $k$ ), but the states of components $\left\{\lambda_{1}, \ldots, \lambda_{s}\right\}$ are fixed. The states of components $\left\{\lambda_{s+1}, \ldots, \lambda_{n}\right\}$ are also fixed in any attractor, to arbitrary values 
among $\{0,1\}$. As $v$ satisfies $\psi$ for any valuation $v^{\prime}:\left\{\lambda_{s+1}, \ldots, \lambda_{n}\right\} \rightarrow\{0,1\}$, the states of components $\left\{C_{1}, \ldots, C_{m}\right\}$ and $\psi$ are all fixed to 1 in any attractor. Hence, in any attractor we have:

- $\Omega$ flips its state at each time step,

- $\psi$ is fixed to state 1.

The local functions of components $\left\{\psi_{0}, \ldots, \psi_{k-1}\right\}$ are designed to prevent any limitcycle of length $k$ in this case: each of them is of the form $f_{\psi_{i}}(x)=x_{\psi_{i}}$, i.e. fixed. As a conclusion any attractor is in a limit-cycle of length $2 \neq k$.

For the reverse direction we consider the contrapositive, suppose that there is no assignment $v:\left\{\lambda_{1}, \ldots, \lambda_{s}\right\} \rightarrow\{0,1\}$ such that all assignments $v^{\prime}:\left\{\lambda_{s+1}, \ldots, \lambda_{n}\right\} \rightarrow$ $\{0,1\}$ verify $\psi[v]\left[v^{\prime}\right] \equiv 1$. From what precedes, for any block-sequential update schedule $W$ there exists a configuration $x$ part of an attractor, with $x_{\lambda_{s+1}}, \ldots, x_{\lambda_{n}}$ chosen such that the state of $\psi$ is fixed to 0 . Without loss of generality let use set $x_{\Omega}=1$. Recall that in any attractor the states of components $\{\Omega\} \cup\left\{\lambda_{1}^{\prime}, \ldots, \lambda_{s}^{\prime}\right\} \cup\left\{\lambda_{1}^{\prime \prime}, \ldots, \lambda_{s}^{\prime \prime}\right\}$ flip at each time step, the states of components $\left\{\lambda_{1}, \ldots, \lambda_{n}\right\} \cup\left\{C_{1}, \ldots, C_{m}\right\}$ are fixed, and that $k$ is even. Now if we let $x_{\psi_{0}}=x_{\psi_{1}}=1$ and $x_{\psi_{2}}=\cdots=x_{\psi_{k-1}}=0$, then we claim that $x$ is in a limit-cycle of length $k$. We have to consider that in $W$, each $\psi_{i}$ may either be updated before $\Omega$, or strictly after $\Omega$, and we also have to consider the parity of $i$. According to local functions $f_{\psi_{i}}$, and because the state of component $\psi$ is fixed to 1 , the four cases are as follows (recall that initially $x_{\Omega}=1$ ):

- if $i$ is even and $\psi_{i}$ is updated before $\Omega$, then component $\psi_{i}$ copies the state of $\psi_{i-1} \bmod k$ at even time steps and is unchanged at odd time steps,

- if $i$ is even and $\psi_{i}$ is updated strictly after $\Omega$, then component $\psi_{i}$ copies the state of $\psi_{i-1} \bmod k$ at odd time steps and is unchanged at even time steps,

- if $i$ is odd and $\psi_{i}$ is updated before $\Omega$, then component $\psi_{i}$ copies the state of $\psi_{i-1}$ at odd time steps and is unchanged at even time steps,

- if $i$ is odd and $\psi_{i}$ is updated strictly after $\Omega$, then component $\psi_{i}$ copies the state of $\psi_{i-1}$ at even time steps and is unchanged at odd time steps.

Now observe that in any case, thanks to the parity of $i$ and the order of $\psi_{i}$ relative to component $\Omega$, when $\psi_{i}$ copies the state of $\psi_{i-1} \bmod k$, it is not possible that $\psi_{i-1} \bmod k$ has already copied the state of $\psi_{i-2} \bmod k$. As a consequence, at each time step the couple of states 1 moves one component forward along the cycle $\left\{\psi_{0}, \ldots, \psi_{k-1}\right\}$, and after $k$ time steps we have $\left(f^{(W)}(x)\right)^{k}=x$ (and not before).

In the construction above, the fact that $f_{\Omega}(x)=\neg x_{\Omega}$ imposes that any configuration converges to a limit-cycle of even length. Component $\Omega$ acts as a clock. For $k=2$ we can adapt the construction by letting $x_{\psi}$ stop this clock when the formula is satisfied, then in this case any configuration converges to a fixed point.

Corollary 2. BS no $k$-LC is NP ${ }^{N P}$-hard for $k=2$.

Proof. We present again a reduction from $\exists \forall$-3-SAT, with a slightly modified construction from Theorem 3. Given a 3 -CNF formula $\psi$ on $\left\{\lambda_{1}, \ldots, \lambda_{n}\right\}$ with $m$ clauses denoted 
as usual $C_{1}, \ldots, C_{m}$, and an integer $s \in[n]$, we construct the following $\mathrm{BN}$ of size $2 s+n+m+2$. The components are

$$
\{\Omega, \psi\} \cup\left\{\lambda_{1}, \ldots, \lambda_{n}\right\} \cup\left\{\lambda_{1}^{\prime}, \ldots, \lambda_{s}^{\prime}\right\} \cup\left\{\lambda_{1}^{\prime \prime}, \ldots, \lambda_{s}^{\prime \prime}\right\} \cup\left\{C_{1}, \ldots, C_{m}\right\}
$$

and the local functions are

- $f_{\Omega}(x)=\neg x_{\Omega} \wedge \neg x_{\psi}$,

- $f_{\lambda_{i}^{\prime}}(x)=f_{\lambda_{i}^{\prime \prime}}(x)=x_{\Omega}$ for $i \in[s]$,

- $f_{\lambda_{i}}(x)=x_{\lambda_{i}^{\prime}} \oplus x_{\lambda_{i}^{\prime \prime}}$ for $i \in[s]$, and $f_{\lambda_{i}}(x)=x_{\lambda_{i}}$ for $i \in[n] \backslash[s]$,

- $f_{C_{j}}(x)=\bigvee_{\lambda_{i} \in C_{j}} x_{\lambda_{i}} \vee \bigvee \neg \lambda_{i} \in C_{j} \neg x_{\lambda_{i}}$ for $j \in[m]$,

- $f_{\psi}(x)=\left(x_{C_{1}} \wedge \cdots \wedge x_{C_{m}}\right) \vee x_{\psi}$.

In this construction, the valuation of existential variables is still encoded in the block-sequential update schedule $W$, and all combinations of states on components corresponding to universal variables still appear in attractors. Now if the formula $\psi$ is a negative instance of $\exists \forall-\mathbf{3 - S A T}$, then for any $W$ there exists a complete valuation (existential and universal variables) not satisfying the formula, hence in some attractor we have $x_{\psi}=0$, and component $\Omega$ flips at each step, giving a limit-cycle of length 2 . On the contrary, if $\psi$ is a positive instance of $\exists \forall$-3-SAT, then there exists a $W$ such that all complete valuations satisfy the formula, hence in all attractors we have $x_{\psi}=1$ (suppose $x_{\psi}=0$, then it will converge to state 1 under update schedule $W$ ). Finaly, if $x_{\psi}=1$ then the attractor is a fixed point (it fixes component $\Omega$, then $\lambda_{i}^{\prime \prime}, \lambda_{i}^{\prime}, \lambda_{i}$, then $C_{j}$ ), thus in this case there is no limit-cycle of length other than 1 .

The idea presented in Corollary 2 of stopping a clock when the formula is satisfied (the clock gives a limit-cycle of length $k$, and stopping it leads to a fixed point), can be extended to any $k>2$. The challenge here is to design a clock giving a limit-cycle of length $k$ for any block-sequential update schedule.

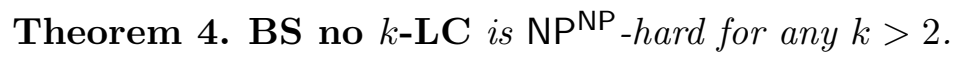

Proof. The reduction is again from $\exists \forall-3-S A T$. Given a 3 -CNF formula $\psi$ on $\left\{\lambda_{1}, \ldots, \lambda_{n}\right\}$ with $m$ clauses denoted as usual $C_{1}, \ldots, C_{m}$, and an integer $s \in[n]$, we construct a BN of size $s+n+m+k+\left\lceil\log _{2}\left(\mathrm{BS}_{k+1}\right)\right\rceil+3$ with $\mathrm{BS}_{k}$ the number of block-sequential update schedules ${ }^{3}$ of size $k$, on the components

$$
\begin{gathered}
\left\{\Omega_{0}, \ldots, \Omega_{k}\right\} \cup\left\{\omega_{1}, \ldots, \omega_{\left\lceil\log _{2}\left(\mathrm{BS}_{k+1}\right)\right\rceil}\right\} \cup\{\text { stop }\} \\
\cup\left\{\lambda_{1}, \ldots, \lambda_{n}\right\} \cup\left\{\lambda_{1}^{\prime}, \ldots, \lambda_{s}^{\prime}\right\} \cup\left\{C_{1}, \ldots, C_{m}\right\} \cup\{\psi\} .
\end{gathered}
$$

\footnotetext{
3 The number of block-sequential update schedules of size $k$ equals the number of ordered partitions of a set of $k$ elements, also known as ordered Bell number (sequence A000670 in the OEIS [1]). We have

$$
\mathrm{BS}_{n}=\sum_{i=0}^{k} i !\left\{\begin{array}{l}
k \\
i
\end{array}\right\}=\sum_{i=0}^{k} \sum_{j=0}^{i}(-1)^{i-j}\left(\begin{array}{l}
i \\
j
\end{array}\right) j^{k}
$$

using the Stirling numbers of the second kind (denoted with \{\} ) counting the number of surjective maps from a set of $i$ elements to a set of $k$ elements [18.
} 
Recall that $k$ is fixed in the problem definition, hence we do not need ${ }^{4}$ to consider the growth of $\log _{2}\left(\mathrm{BS}_{k+1}\right)$, which is a constant from the point of view of BS no $k$-LC.

The idea is to build a clock of length $k$ on the $k+1$ components $\Omega$, with some 1 state moving forward at each step. However, it will not move forward from components $\Omega_{i}$ to $\Omega_{i+1}$, etc modulo $k$, but instead it will move forward according to the order of components $\Omega$ in the current update schedule, which is supposed to be encoded (in binary $y^{5}$ on components $\omega$ (positive loops on components $\omega$ will let them take any fixed value in attractors). Similarly to the construction of Theorem 3 , the update order of $\lambda_{i}, \lambda_{i}^{\prime}$ compared to clock component $\Omega_{0}$ encodes existential variables in $W$, and positive loops on universal variables let them take any fixed value in attractors. Finally, $x_{\psi}=$ 1 will stop the clock. Regarding the logics of the proof, if $\psi$ is a positive instance then one can choose $W$ with components $\Omega$ updated in parallel and $\lambda_{i}^{\prime}$ encoding the existential variables to satisfy $\psi$, then component $\psi$ will be in state 1 in any attractor (thanks to the construction, regardless of the update schedule encoded on components $\omega)$ hence leading to fixed points only. If $\psi$ is a negative instance, then for any $W$ we can set components $\omega$ accordingly to have a working clock of length $k$, and no matter the encoding of existential variables there exists a choice of states on components corresponding to universal variables such that $\psi$ is in state 0 , letting the clock tick forever and create a limit-cycle of length $k$.

The local functions are

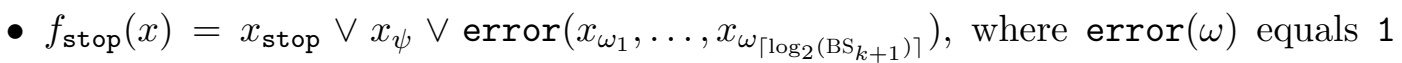
when components $\omega$ do not encode a block-sequential update schedule,

- $f_{\omega_{i}}(x)=x_{\omega_{i}}$ for $i \in\left[\left\lceil\log _{2}\left(\mathrm{BS}_{k+1}\right)\right\rceil\right]$,

- for the definition of $\Omega_{i}$, let us consider the update schedule encoded on components $\omega$ in some configuration $x$, and denote $j_{0}(x), \ldots, j_{k}(x)$ the lexicographically minimal permutation of $0, \ldots, k$ such that $\Omega_{j_{0}(x)} \preccurlyeq x_{\omega} \Omega_{j_{1}(x)} \preccurlyeq x_{\omega} \cdots \preccurlyeq x_{\omega} \Omega_{j_{k}(x)}$, where $a \preccurlyeq x_{\omega} b$ means that component $a$ is updated prior to or simultaneously with component $b$ in the update schedule encoded on components $\omega$ in configuration $x$; for $i \in\{0, \ldots, k\}, f_{\Omega_{i}}(x)=\neg x_{\text {stop }} \wedge\left\{\begin{array}{c}1 \quad \text { if } i=j_{p}(x) \text { and } x_{\Omega_{j_{p-1}(x)}}=1 \text { and } \\ \left(x_{\omega} \neq\left(\left\{\Omega_{0}, \ldots, \Omega_{k}\right\}\right) \text { or } i \neq k\right) \\ 0 \quad \text { otherwise, }\end{array}\right.$ with $x_{\omega}$ the block-sequential update schedule encoded on components $\omega$,

- $f_{\lambda_{i}^{\prime}}(x)=x_{\Omega_{0}}$ for $i \in[s]$,

- for $i \in[s], f_{\lambda_{i}}(x)= \begin{cases}x_{\Omega_{0}} \oplus x_{\lambda_{i}^{\prime}} & \text { if } x_{\Omega_{0}}=1 \\ x_{\lambda_{i}} & \text { otherwise, }\end{cases}$ and for $i \in[n] \backslash[s], f_{\lambda_{i}}(x)=x_{\lambda_{i}}$,

- $f_{C_{j}}(x)=\bigvee_{\lambda_{i} \in C_{j}} x_{\lambda_{i}} \vee \bigvee \neg \lambda_{i} \in C_{j} \neg x_{\lambda_{i}}$ for $j \in[m]$,

- $f_{\psi}(x)=\left(x_{C_{1}} \wedge \cdots \wedge x_{C_{m}}\right)$.

\footnotetext{
4 TODO: Can we nevertheless consider it, just for fun, in this footnote?

5 Since $k$ is a constant we can consider any computable encoding of the block-sequential update schedules, for example their numbering according to the lexicographic order (each subset of $\left\{\Omega_{0}, \ldots, \Omega_{k}\right\}$ corresponds to a digit on $k$ bits).
} 


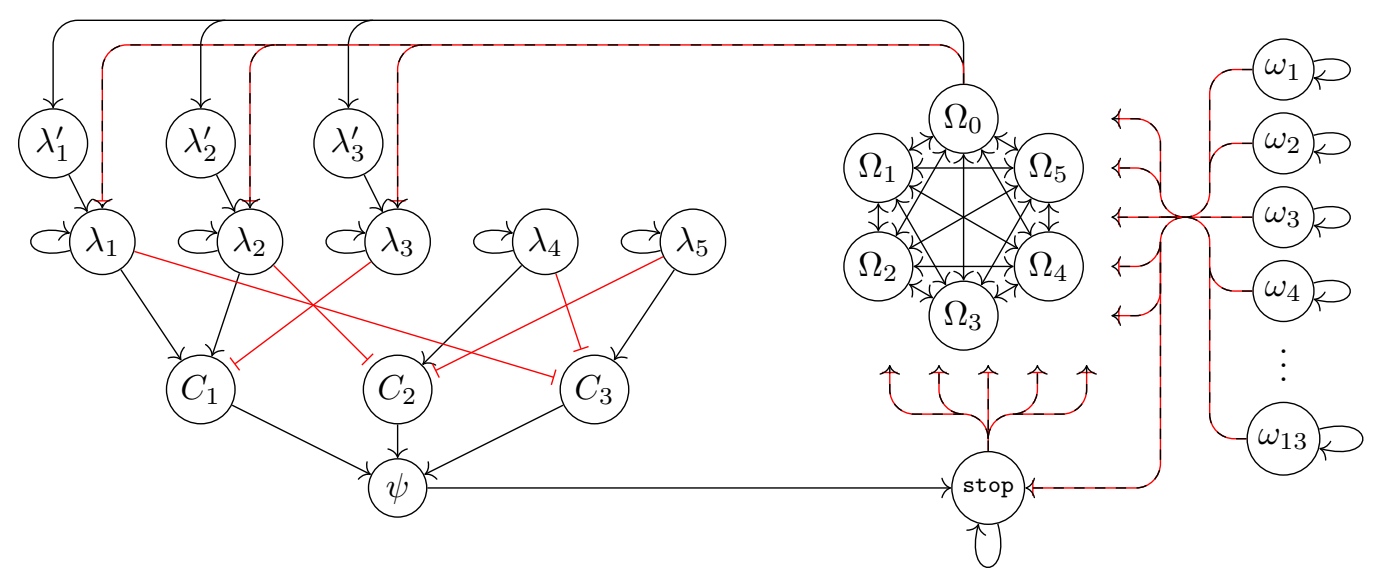

Figure 4: Signed interaction digraph of the BN obtained for $k=5\left(\mathrm{BS}_{6}=4683\right)$, the 3-CNF formula $\psi=\left(\lambda_{1} \vee \lambda_{2} \vee \neg \lambda_{3}\right) \wedge\left(\neg \lambda_{2} \vee \lambda_{4} \vee \neg \lambda_{5}\right) \wedge\left(\neg \lambda_{1} \vee \neg \lambda_{4} \vee \lambda_{5}\right)$, and $s=3$. Negative arcs $(-)$ are red with a flat head, positive arcs $(+)$ are black, positive-negative $\operatorname{arcs}( \pm)$ are dashed with both colors and heads. All components stop and $\omega_{1}, \ldots, \omega_{13}$ are connected to all components $\Omega_{0}, \ldots, \Omega_{5}$ with arcs of sign \pm .

An example signed interaction digraph of this BN is presented on Figure 4. First remark that if $x_{\text {stop }}=1$ then $x$ converges to a fixed point (the clock stops), hence we will consider thereafter only attractors from configurations with component stop in state 0 .

Suppose $\psi$ is a negative instance of $\exists \forall$-3-SAT. For any block-sequential update schedule $W$, consider a configuration $x$ such that components $\omega$ encode the projection of $W$ on the clock components. If $W$ is not the parallel update schedule, the clock has the following dynamics (time goes downward, one step per line):

\begin{tabular}{|c|c|c|c|c|c|c|}
\hline$\Omega_{j_{k}(x)}$ & $\Omega_{j_{k-1}(x)}$ & $\Omega_{j_{k-2}(x)}$ & $\ldots$ & $\Omega_{j_{2}(x)}$ & $\Omega_{j_{1}(x)}$ & $\Omega_{j_{0}(x)}$ \\
\hline 0 & 1 & 0 & $\ldots$ & 0 & 0 & 0 \\
\hline 0 & 0 & 1 & $\ldots$ & 0 & 0 & 0 \\
\hline$\vdots$ & $\vdots$ & $\vdots$ & & $\vdots$ & $\vdots$ & $\vdots$ \\
\hline 0 & 0 & 0 & $\ldots$ & 1 & 0 & 0 \\
\hline 0 & 0 & 0 & $\ldots$ & 0 & 1 & 0 \\
\hline 1 & 0 & 0 & $\ldots$ & 0 & 0 & 1 \\
\hline 0 & 1 & 0 & $\ldots$ & 0 & 0 & 0 \\
\hline
\end{tabular}

and if $W$ is the parallel update schedule $\left(\left\{\Omega_{0}, \ldots, \Omega_{k}\right\}\right)$, the clock has the following 
dynamics (time goes downward, one step per line):

\begin{tabular}{|c|c|c|c|c|c|c|}
\hline$\Omega_{0}$ & $\Omega_{1}$ & $\Omega_{2}$ & $\ldots$ & $\Omega_{k-2}$ & $\Omega_{k-1}$ & $\Omega_{k}$ \\
\hline 0 & 1 & 0 & $\ldots$ & 0 & 0 & 0 \\
\hline 0 & 0 & 1 & $\ldots$ & 0 & 0 & 0 \\
\hline$\vdots$ & $\vdots$ & $\vdots$ & & $\vdots$ & $\vdots$ & $\vdots$ \\
\hline 0 & 0 & 0 & $\ldots$ & 1 & 0 & 0 \\
\hline 0 & 0 & 0 & $\ldots$ & 0 & 1 & 0 \\
\hline 1 & 0 & 0 & $\ldots$ & 0 & 0 & 0 \\
\hline 0 & 1 & 0 & $\ldots$ & 0 & 0 & 0 \\
\hline
\end{tabular}

thus we have a clock of length $k$ in any case:

- when $W$ is not parallel the minimum component according to $\preccurlyeq x_{\omega}$ and the lexicographical order is skipped (the 1 state moves two components forward),

- when $W$ is parallel component $\Omega_{k}$ is discarded (it remains in state 0 and the clock ticks on components $\left.\Omega_{0}, \ldots, \Omega_{k-1}\right)$.

Furthermore, for $i \in[s]$ component $\lambda_{i}^{\prime}$ goes to state 1 exactly once every $k$ steps, and the relative positions of components $\lambda_{i}^{\prime}, \lambda_{i}, \Omega_{0}$ fixes the value of component $\lambda_{i}$ :

- if $\left(\lambda_{i}^{\prime}=_{W} \Omega_{0}\right)$ or $\left(\Omega_{0} \prec_{W} \lambda_{i} \preccurlyeq_{W} \lambda_{i}^{\prime}\right)$ or $\left(\lambda_{i} \preccurlyeq_{W} \lambda_{i}^{\prime} \prec_{W} \Omega_{0}\right)$ or $\left(\lambda_{i}^{\prime} \prec_{W} \Omega_{0} \prec_{W} \lambda_{i}\right)$ then $x_{\lambda_{i}}=1$,

- otherwise $x_{\lambda_{i}}=0$.

Since the instance $\psi$ is negative, for any assignment of states to components $\lambda_{1}, \ldots, \lambda_{s}$ (corresponding to existential variables), we can set the states of components $\lambda_{s+1}, \ldots, \lambda_{n}$ (corresponding to universal variables) so that at least one clause $C_{j}$ is not satisfied hence $x_{C_{j}}=0$ and $x_{\psi}=0$. As a consequence $f_{\text {stop }}(x)=0$, i.e. the clock is not stopped, and therefore it creates a limit-cycle of length $k$.

Suppose $\psi$ is a positive instance of $\exists \forall$-3-SAT, with $v:\left\{\lambda_{1}, \ldots, \lambda_{s}\right\} \rightarrow\{0,1\}$ an assignment such that for all $v^{\prime}:\left\{\lambda_{s+1}, \ldots, \lambda_{n}\right\} \rightarrow\{0,1\}$ we have $\psi[v]\left[v^{\prime}\right] \equiv 1$. We define

$$
W=\left(T^{\prime},\left\{\Omega_{0}, \ldots, \Omega_{k}\right\}, \mathcal{R}\right),
$$

with $T^{\prime}=\left\{\lambda_{i}^{\prime} \mid v\left(\lambda_{i}\right)=1\right\}$ and $\mathcal{R}$ all the other components. We consider a case disjunction on the starting configuration.

- If components $\omega$ encode the parallel update schedule, then from what preceeds the states of components $\lambda_{i}$ for $i \in[s]$ encode $v$ and component $\psi$ will eventually be in state 1, so does component stop and the clock stops, leading to a fixed point.

- If components $\omega$ do not encode the parallel update schedule, then from the definition of local function $f_{\Omega_{i}}$ we will have a clock of length $k+1$ (with state 1 moving one component forward at each step, in an order given by the update schedule encoded on components $\omega$ ). However, it does not alter the fact that the states of components $\lambda_{i}$ for $i \in[s]$ encode $v$, therefore the same deductions apply: the configuration converges to a fixed point.

We can conclude that under update schedule $W$, any configuration converges to a fixed point hence there is no limit-cycle of length $k$. 
Remark 3. Encoding local function as truth tables of the components it effectively depends on (its in-neighbors in the interaction digraph) would also lead to the same complexity results, because all the constructions presented for hardness results can be adapted so that each component depends on a bounded number of components (the resulting interaction digraph has a bounded in-degree), given that $k$ is a constant.

\section{Conclusion}

We have characterized precisely the computational complexity of problems related to, given a $\mathrm{BN}$, the existence or not of limit-cycles of some fixed length $k$, with the quantifier alternation of "does there exist an update schedule such that all configurations are not in a limit-cycle of size $k$ " bringing us to level $\Sigma_{2}^{\mathrm{P}}$ of the polynomial hierarchy.

Remark that all the constructions presented in our reductions (except for Theorem ?? which is subsumed by Theorem ??) are such that the resulting BN has either some limit cycles of size $k$, or only fixed points. Consequently, the same results directly hold for the problem $\phi_{k}\left(f^{(W)}\right)$ is replaced by $\phi_{\geq k}\left(f^{(W)}\right)=\sum_{\ell \geq k} \phi_{\ell}\left(f^{(W)}\right)$, i.e. we consider limitcycles of length at least $k$ instead of exactly $k$. With little additional work the proofs may also be adapted to $\phi_{\leq k}\left(f^{(W)}\right)=\sum_{\ell \leq k} \phi_{\ell}\left(f^{(W)}\right)$, i.e. if we consider limit-cycles of length at most $k$ (fixed points should be transformed into limit-cycles of length larger that $k)$.

Finally, if $k$ is part of the input, is there a drastic complexity increase as observed for problems related to the number of fixed points in [6]? The construction presented in the proof of Theorem 4 makes heavy use of being a $k$ constant.

We hope that these first results on the complexity of deciding the existence of limitcycles in Boolean networks opens a promising research direction, confronting the necessary difficulty of considering a diversity of update schedules. The lens of computational complexity reveals, via the gadgets employed in lower bound constructions, mechanisms at the heart of Boolean network's dynamical richness.

\section{Acknowledgments}

The authors are thankful to project ANR-18-CE40-0002-01 "FANs", project ECOSCONICYT C16E01, project STIC AmSud CoDANet 19-STIC-03 (Campus France 43478PD), for their funding.

\section{References}

[1] OEIS A000679. The online encyclopedia of integer sequences. https://oeis.org/ A000670.

[2] J. Aracena, J. Demongeot, É. Fanchon, and M. Montalva. On the number of different dynamics in Boolean networks with deterministic update schedules. Math. Biosci., 242:188-194, 2013. 
[3] J. Aracena, J. Demongeot, and E. Goles. Fixed points and maximal independent sets in AND-OR networks. Discr. Appl. Math., 138:277-288, 2004.

[4] J. Aracena, L. Gómez, and L. Salinas. Limit cycles and update digraphs in Boolean networks. Disc. Appl. Math., 161:1-12, 2013.

[5] J. Aracena, A. Richard, and L. Salinas. Number of fixed points and disjoint cycles in monotone Boolean networks. SIAM J. Discr. Math., 31:1702-1725, 2017.

[6] F. Bridoux, N. Durbec, K. Perrot, and A. Richard. Complexity of maximum fixed point problem in Boolean networks. In Proc. of CiE'2019, volume 11558 of LNCS, pages 132-143, 2019.

[7] J. Demongeot, M. Noual, and S. Sené. Combinatorics of Boolean automata circuits dynamics. Discr. Appl. Math., 160:398-415, 2012.

[8] B. Elspas. The theory of autonomous linear sequential networks. IRE Trans. Circuit Theory, 6:45-60, 1959.

[9] P. Floreen and P. Orponen. On the computational complexity of analyzing Hopfield nets. Complex Systems, 3:577-587, 1989.

[10] E. Goles and S. Martínez. Neural and automata networks: dynamical behavior and applications. Kluwer Academic Publishers, 1990.

[11] E. Goles and M. Noual. Block-sequential update schedules and Boolean automata circuits. In Proc. of AUTOMATA'2010, DMTCS, pages 41-50, 2010.

[12] L. Gómez. Dynamics of discrete networks with deterministic updates schedules. Application to genetic regulatory networks. PhD thesis, Univ. Concepción, 2015.

[13] D. A. Huffman. Canonical forms for information-lossless finite-state logical machines. IRE Trans. Inform. Theory, 5:41-59, 1959.

[14] R. M. Karp. Reducibility among combinatorial problems. Complexity of Computer Computations, pages 85-103, 1972.

[15] S. A. Kauffman. Homeostasis and differentiation in random genetic control networks. Nature, 224:177-178, 1969.

[16] S. C. Kleene. Representation of events in nerve nets and finite automata. Project RAND RM-704, US Air Force, 1951.

[17] W. S. McCulloch and W. Pitts. A logical calculus of the ideas immanent in nervous activity. J. Math. Biophys., 5:115-133, 1943.

[18] M. Noual and S. Sené. Towards a theory of modelling with Boolean automata networks - I. Theorisation and observations. arXiv:1111.2077, 2011. 
[19] P. Orponen. Neural networks and complexity theory. In Proc. of MFCS'1992, volume 629 of $L N C S$, pages 50-61, 1992.

[20] C. H. Papadimitriou. Computational complexity. Addison-Wesley, 1994.

[21] É. Remy, P. Ruet, and D. Thieffry. Graphic requirements for multistability and attractive cycles in a Boolean dynamical framework. Adv. Appl. Math., 41:335-350, 2008 .

[22] A. Richard. Negative circuits and sustained oscillations in asynchronous automata networks. Adv. Appl. Math., 44:378-392, 2010.

[23] A. Richard and J.-P. Comet. Necessary conditions for multistationarity in discrete dynamical systems. Discr. Appl. Math., 155:2403-2413, 2007.

[24] F. Robert. Blocs-H-matrices et convergence des méthodes itératives classiques par blocs. Linear Algebra Appl., 2:223-265, 1969.

[25] F. Robert. Itérations sur des ensembles finis et automates cellulaires contractants. Linear Algebra Appl., 29:393-412, 1980.

[26] F. Robert. Discrete iterations: a metric study. Springer, 1986.

[27] R. Thomas. Boolean formalization of genetic control circuits. J. Theor. Biol., 42:563-585, 1973.

[28] R. Thomas. On the relation between the logical structure of systems and their ability to generate multiple steady states or sustained oscillations. In Numerical methods in the study of critical phenomena, pages 180-193. Springer, 1981. 